\title{
Increased white matter diffusivity associated with phantom limb pain
}

\author{
Cheong Hoon Seo ${ }^{1, *}$, Chang-hyun Park ${ }^{2, *}$, Myung Hun Jung ${ }^{3}$, Seungki Baek ${ }^{4}$, Jimin Song ${ }^{4}$, Eunsil Cha ${ }^{4}$, and \\ Suk Hoon $\mathrm{Ohn}^{4}$
}

'Department of Physical Medicine and Rehabilitation, Hallym University Hangang Sacred Heart Hospital, Hallym University College of Medicine, Seoul, Korea

${ }^{2}$ Center for Neuroprosthetics and Brain Mind Institute, Swiss Federal Institute of Technology (EPFL), Geneva, Switzerland

${ }^{3}$ Department of Psychiatry, Hallym University Sacred Heart Hospital, Hallym University College of Medicine, Anyang, Korea

${ }^{4}$ Department of Physical Medicine and Rehabilitation, Hallym University Sacred Heart Hospital, Hallym University College of Medicine, Anyang, Korea

Received March 4, 2019

Revised May 30, 2019

Accepted May 30, 2019

\section{Correspondence}

Suk Hoon Ohn

Department of Physical Medicine and

Rehabilitation, Hallym University Sacred

Heart Hospital, 22 Gwanpyeong-ro 170

beon-gil, Dongan-gu, Anyang 14068, Korea

Tel: +82-31-380-3860

Fax: +82-31-380-3864

E-mail: ohneum@gmail.com

*These authors contributed equally to this work.

Key Words

Amputation

Brain

Chronic Pain

Diffusion Tensor Imaging

Magnetic Resonance Imaging

Neuronal Plasticity

Phantom limb

White Matter
Background: We utilized diffusion tensor imaging (DTI) to evaluate the cerebral white matter changes that are associated with phantom limb pain in patients with unilateral arm amputation. It was anticipated that this would complement previous research in which we had shown that changes in cerebral blood volume were associated with the cerebral pain network.

Methods: Ten patients with phantom limb pain due to unilateral arm amputation and sixteen healthy age-matched controls were enrolled. The intensity of phantom limb pain was measured by the visual analogue scale (VAS) and depressive mood was assessed by the Hamilton depression rating scale. Diffusion tensor-derived parameters, including fractional anisotropy, mean diffusivity, axial diffusivity (AD), and radial diffusivity (RD), were computed from the DTI.

Results: Compared with controls, the cases had alterations in the cerebral white matter as a consequence of phantom limb pain, manifesting a higher AD of white matter in both hemispheres symmetrically after adjusting for individual depressive moods. In addition, there were associations between the RD of white matter and VAS scores primarily in the hemispheres related to the missing hand and in the corpus callosum.

Conclusions: The phantom limb pain after unilateral arm amputation induced plasticity in the white matter. We conclude that loss of white matter integrity, particularly in the hemisphere connected with the missing hand, is significantly correlated with phantom limb pain.

\section{INTRODUCTION}

Cerebral gray and white matter lesions may have common etiologies. Dysfunction from vascular [1], degenerative [2], traumatic [3], and aging [4] processes are typically involved in changes to the gray matter and the white matter that provide intracerebral connectivity between primary and secondary lesions. All cerebral dysfunctions can, fundamentally, be understood as consequences of altered neural networks and connectivity. Patients with limb amputation lose afferentation from the missing limb, affecting many brain networks. Indeed, phantom limb pain following such amputation can be understood in the context of a reorganization of the pain network. (c) This is an open-access article distributed under the terms of the Creative Commons Attribution Non-Commercial License (http://creativecommons.org/licenses/by-nc/4.0/), which permits unrestricted non-commercial use, distribution, and reproduction in any medium, provided the original work is properly cited.

(C) The Korean Pain Society, 2019
Author contributions: Cheong Hoon Seo: Writing/manuscript preparation; Chang-hyun Park: Writing/manuscript preparation; Myung Hun Jung: Methodology; Seungki Baek: Investigation; Jimin Song: Investigation; Eunsil Cha: Investigation; Suk Hoon Ohn: Funding acquisition. 
To date, several functional and structural reorganizations of the cortices and the thalamus have been identified as possible mechanisms underlying phantom limb pain [5-9]. Previous neuroimaging studies have revealed that alterations in pain network neural activity occur in both the sensory-perceptive $[7,9]$ and emotional domains [5], both of which play different roles in the experience of pain. The sensory-perceptive domain of the pain network is responsible for pain sensation in the presence of noxious body stimuli and involves the thalamus, primary somatosensory cortex, secondary somatosensory cortex, and insula. The emotional domain of the pain network is responsible for reacting to pain with negative emotional events and memories and involves the orbitofrontal cortex (OFC), anterior cingulate cortex (ACC), amygdala, and insula [10]. We also noted that increased cerebral blood volume (CBV) in the ACC and the OFC reflected a functional reorganization of the emotion-related pain network in patients with phantom limb pain [11].

Diffusion tensor imaging (DTI) is an advanced magnetic resonance imaging (MRI) technique that can identify alterations to the white matter tract and quantifies these changes according to fractional anisotrophy (FA), mean diffusivity (MD), axial diffusivity (AD), and radial diffusivity (RD) indices. Pathologic findings on white matter injury, whether axonopathy or myelinopathy, also can be inferred from DTI indices [12,13]. We hypothesized that patients with phantom limb pain after amputation would have changes not only in the gray matter but also in the white matter. In the present study, we therefore aimed to analyze DTI data to identify the white matter link to the pain network in the same subjects who participated in our previous CBV study [11].

\section{MATERIALS AND METHODS}

\section{Participants}

This was an exploratory case-control study. We used the DTI data acquired at the time of CBV MRI in our previous study [11]. Thus, ten patients with phantom limb pain (43.8 \pm $3.4 \mathrm{yr}$, range: $37-48 \mathrm{yr}, 1$ woman) and sixteen age- and sexmatched healthy controls $(41.7 \pm 2.2 \mathrm{yr}$, range: $39-46 \mathrm{yr}$, 7 women) participated. The mean duration of phantom limb pain was $44.8 \pm 35.1$ months (range: $16-115 \mathrm{mo}$ ). Their demographic and clinical data are summarized in Table 1. Written informed consent was obtained from all participants in accordance with the Declaration of Helsinki and its later amendments, and the Institutional Review Board of Hallym University Sacred Heart Hospital approved the study (IRB No. 2013-106, 2014-I079).

Participants were required to meet the following inclusion criteria: (1) were aged less than 50 years, (2) had undergone amputation of a unilateral upper limb after electrical injury, (3) had suffered from phantom limb pain for longer than 12 months, and (4) had uncontrolled pain despite medication and physical therapy. Participants were excluded from the study if any of the following criteria were met: (1) phantom limb sensation, (2) residual limb pain, (3) pain resulting from other causes and confirmed by imaging, (4) other forms of persistent pain lasting more than three months, (5) a history of cardiac arrest due to electrical injury, (6) a history of brain disease or surgery, (7)

Table 1. Demographic and Clinical Characteristics of Participants

\begin{tabular}{|c|c|c|c|c|c|c|c|}
\hline Variable & Age (yr) & Sex & $\begin{array}{c}\text { Upper limb } \\
\text { amputation (\%) }\end{array}$ & $\begin{array}{l}\text { Duration of phantom } \\
\text { limb pain (mo) }\end{array}$ & $\begin{array}{l}\text { VAS of phantom } \\
\text { limb pain }\end{array}$ & HDRS & MMSE-K \\
\hline \multirow[t]{11}{*}{ Patients $(n=10)$} & 43 & M & 100 & 40 & 10 & 25 & 30 \\
\hline & 44 & M & 9 & 19 & 7 & 27 & 28 \\
\hline & 48 & M & 46 & 22 & 5 & 23 & 23 \\
\hline & 45 & M & 41 & 19 & 3 & 18 & 28 \\
\hline & 39 & M & 43 & 18 & 4 & 17 & 29 \\
\hline & 47 & M & 45 & 115 & 7 & 0 & 28 \\
\hline & 44 & M & 17 & 16 & 7 & 30 & 30 \\
\hline & 45 & $\mathrm{~F}$ & 94 & 61 & 7 & 26 & 27 \\
\hline & 46 & M & 43 & 95 & 3 & 6 & 27 \\
\hline & 37 & $M$ & 12 & 43 & 7 & 23 & 28 \\
\hline & $43.8 \pm 3.4$ & $9: 1^{a}$ & $45.0 \pm 30.1$ & $44.8 \pm 35.1$ & $6.0 \pm 2.2$ & $19.5 \pm 9.6$ & $27.8 \pm 2.0$ \\
\hline Controls $(n=16)$ & $41.7 \pm 2.2$ & $9: 7^{a}$ & N/A & N/A & N/A & $2.9 \pm 3.6$ & $28.9 \pm 1.6$ \\
\hline$P$ value & 0.103 & 0.352 & & & & $<0.001$ & 0.145 \\
\hline
\end{tabular}

Values are presented as number only or mean \pm standard deviation.

VAS: visual analogue scale, HDRS: Hamilton depression rating scale, MMSE-K: mini mental state examination (Korean version), M: male, F: female, N/A: not applicable.

${ }^{\mathrm{a} M: F .}$ 
the presence of a cardiac pacemaker, and (8) pregnancy.

\section{Clinical assessment}

The percentage of amputation was calculated using the following formula:

(non-amputated arm length - residual limb length) /

(non-amputated arm length) $\times 100$

where the non-amputated arm length was defined as the distance between the acromion and third fingertip of the non-amputated arm. Participants were asked to describe the average intensity of phantom limb pain over the last week using the visual analogue scale (VAS). Depressive mood was assessed using the previously validated Korean version of the Hamilton depression rating scale (HDRS) [14]. Cognitive function was assessed using the Korean version of the Mini Mental State Examination (MMSE-K). Both HDRS and MMSE-K were administered to all participants by a single licensed psychologist. VAS, HDRS, and MMSE-K assessments were administered on the same day that the MRI was obtained.

\section{Acquisition and analysis of DTI data}

Diffusion-weighted MRI data were obtained using a 1.5T MRI scanner (MAGNETOM Sonata ${ }^{\mathrm{TM}}$; Siemens, Erlangen, Germany). For each participant, 13 images comprising 12 images with high diffusion weighting ( $b$ value $=1,000 \mathrm{sec}$ / $\mathrm{mm}^{2}$ ) and one with no diffusion weighting ( $b$ value $=0 \mathrm{sec} /$ $\mathrm{mm}^{2}$ ) were acquired with a single-shot diffusion- weighted echo planar imaging sequence. Each image included 39 axial slices with the following parameters: repetition time, 6,600 msec; echo time, 96 msec; section thickness, $4.4 \mathrm{~mm}$; no intersection gap; plane resolution, $1.8 \mathrm{~mm} \times 1.8 \mathrm{~mm}$; matrix, $128 \times 128$; field of view, $230 \mathrm{~mm} \times 230 \mathrm{~mm}$.

Preprocessing and diffusion tensor modeling were performed with the FDT v3.0 software that was included in the functional MRI of the brain (FMRIB) software library (FSL; http://fsl.fmrib.ox.ac.uk/fsl/). Each of the participants' 13 images was first realigned to the image with no diffusion weighting to correct for distortions induced by eddy currents and simple head motion. At each voxel within the whole-brain mask created by excluding nonbrain tissue, we fit a diffusion tensor model and computed diffusion tensor-derived parameters (the "derived measures"). These derived measures were the FA, MD, AD, and $\mathrm{RD}$. Given the three diffusivities along different axes of the diffusion tensor, FA was calculated as reflecting diffusivity differences between the three axes; MD, as the average diffusivity across the three axes; $\mathrm{AD}$, as the greatest diffusivity along the principal axis; and $\mathrm{RD}$, as the average of the diffusivities along two minor axes.
We flipped the images used for the derived measures around the mid-sagittal axis for patients who underwent amputation of the left upper limb, allowing the amputated upper limb to be uniformly connected with the left hemisphere in each patient. Then, for voxel-wise statistical analyses of the derived measures, we utilized a tractbased spatial statistics (TBSS) approach [15], which was implemented in FSL. For the FA images of individual participants, TBSS was performed by (1) collecting the images from the two groups, (2) applying nonlinear registration of all images into the standard space (FMRIB58_FA image in FSL), (3) aligning the images to create an alignmentinvariant tract representation (mean FA skeleton), and (4) projecting the FA images of individual participants onto the mean FA skeleton. For the images of the other derived measures, information acquired from nonlinear registration and skeletonization of the FA images was employed, such that the $\mathrm{MD}, \mathrm{AD}$, and $\mathrm{RD}$ images of each participant were projected onto the mean FA skeleton.

\section{Statistical inference}

Age, HDRS, and MMSE-K scores were compared between the groups using independent $t$-tests. Sex distribution was compared between the study groups using Pearson's chisquared test. Spearman correlation tests were used to analyze relationships between pain severity (VAS score) and potentially contributing factors, including age, upper limb amputation percentage, phantom limb pain duration, and HDRS and MMSE-K scores. All statistical analyses of the demographic and clinical data of the participants were conducted using IBM SPSS Statistics ver. 20.0 (IBM Corp., Armonk, NY).

For each derived measure, the images projected onto the mean FA skeleton were fed into voxel-wise analyses to examine differences in values between the patients and controls. Also, for each derived measure, associations between derived and clinical measure values, including the percentage of amputation, duration of phantom limb pain, and VAS scores, were assessed. Given that HDRS scores were much higher in patients than in controls $\left(t_{24}=\right.$ $6.2946, P<0.0001$ ), and given that individual differences in the severity of depression may affect both the white matter microstructure [16] and pain [17], HDRS scores were included as a nuisance covariate in the statistical models. That is, HDRS scores as well as ages and sexes were included as columns of a design matrix for the general linear model, and contrast values of zero were applied to those covariates so that their effects on derived measures were corrected for. Statistical inferences were made by employing the nonparametric permutation method implemented in the randomized routine of FSL [18]. As mass univariate 
analyses were performed for 85,901 voxels on the mean FA skeleton, significant differences and associations were determined via the threshold-free cluster enhancement approach [19] at a $P$ value of 0.05 , corrected for multiple comparisons across the voxels.

\section{RESULTS}

\section{Alterations in the derived measures}

When the HDRS scores were included as a nuisance covariate in the statistical models, patients showed higher $\mathrm{AD}$ values compared with the healthy controls. The white matter structures, associated with the altered AD values in patients, comprised the internal capsule, posterior thalamic radiation, sagittal striatum, corona radiata, external capsule, superior longitudinal fasciculus, superior frontooccipital fasciculus, and fornix in both hemispheres (Fig. 1, Table 2). The distribution of altered white matter structures was symmetric over the two hemispheres: $50.2 \%$ in the hemisphere associated with the missing hand and $49.8 \%$ in the hemisphere associated with the intact hand. There were no differences in the FA, MD, or RD values between the patient and control groups.

\section{Pain correlates of the derived measures}

When the HDRS scores were included as a nuisance covariate, the RD values of patients correlated positively with the VAS scores, specifically across white matter structures such as the corpus callosum (between hemispheres) and the corona radiata, superior longitudinal fasciculus, splenium, cingulum, and posterior thalamic radiation (mainly in the hemisphere associated with the missing hand) (Fig. 2, Table 3). Indeed, when we counted the number of statistically significant voxels included in individual hemispheres, the distribution of white matter structures was weighted toward the hemisphere associated with the missing hand, with $84.5 \%$ in the hemisphere connecting to the missing hand and $15.5 \%$ in the hemisphere connecting to the intact hand. No associations were observed for the other derived measures.

Table 2. Labels of White Matter Structures in Which the Axial Diffusivity Values Were Higher in Patients with Phantom Limb Pain than in Healthy Controls

\begin{tabular}{|c|c|c|c|}
\hline White matter label (M or I) & $\begin{array}{c}\text { Maximum } \\
t \text { value }\end{array}$ & $P$ value & $\begin{array}{c}\text { Ratio } \\
(\%)\end{array}$ \\
\hline Internal capsule: posterior limb (I) & 5.3556 & 0.0302 & 4.39 \\
\hline Internal capsule: retrolenticular part (I) & 5.2337 & 0.0248 & 6.99 \\
\hline Posterior thalamic radiation (I) & 5.1886 & 0.0309 & 1.64 \\
\hline Sagittal stratum (I) & 4.9710 & 0.0297 & 1.06 \\
\hline Posterior corona radiata (I) & 4.5636 & 0.0255 & 3.36 \\
\hline Internal capsule: retrolenticular part (M) & 4.3740 & 0.0375 & 4.95 \\
\hline Superior corona radiata (I) & 4.3461 & 0.0288 & 7.16 \\
\hline Internal capsule: posterior limb (M) & 4.2598 & 0.0375 & 7.38 \\
\hline Internal capsule: anterior limb (I) & 4.1947 & 0.0334 & 5.28 \\
\hline Superior corona radiata $(\mathrm{M})$ & 4.1803 & 0.0318 & 7.50 \\
\hline Anterior corona radiata $(\mathrm{I})$ & 4.1717 & 0.0288 & 5.22 \\
\hline External capsule (I) & 4.1030 & 0.0310 & 4.22 \\
\hline Anterior corona radiata $(\mathrm{M})$ & 3.9630 & 0.0380 & 5.99 \\
\hline Posterior corona radiata (M) & 3.9455 & 0.0387 & 3.41 \\
\hline Cerebral peduncle (M) & 3.6441 & 0.0408 & 0.34 \\
\hline Internal capsule: anterior limb (M) & 3.5877 & 0.0391 & 1.62 \\
\hline Superior longitudinal fasciculus (M) & 3.3355 & 0.0413 & 1.13 \\
\hline External capsule (M) & 3.2983 & 0.0392 & 0.24 \\
\hline Superior fronto-occipital fasciculus (M) & 2.9620 & 0.0340 & 0.17 \\
\hline Superior fronto-occipital fasciculus (I) & 2.8277 & 0.0352 & 0.52 \\
\hline Fornix (I) & 2.7395 & 0.0310 & 0.14 \\
\hline Fornix (M) & 2.5467 & 0.0439 & 0.03 \\
\hline Sagittal stratum (M) & 2.2593 & 0.0471 & 0.27 \\
\hline Posterior thalamic radiation (M) & 2.2510 & 0.0497 & 0.03 \\
\hline Corpus callosum: genu & 2.2090 & 0.0405 & 0.07 \\
\hline Tapetum (I) & 1.9822 & 0.0334 & 0.02 \\
\hline Superior longitudinal fasciculus (I) & 1.9583 & 0.0472 & 0.08 \\
\hline Corpus callosum: splenium & 1.7390 & 0.0448 & 0.12 \\
\hline
\end{tabular}

M: hemisphere associated with missing hand, I: hemisphere asso-ciated with intact hand.

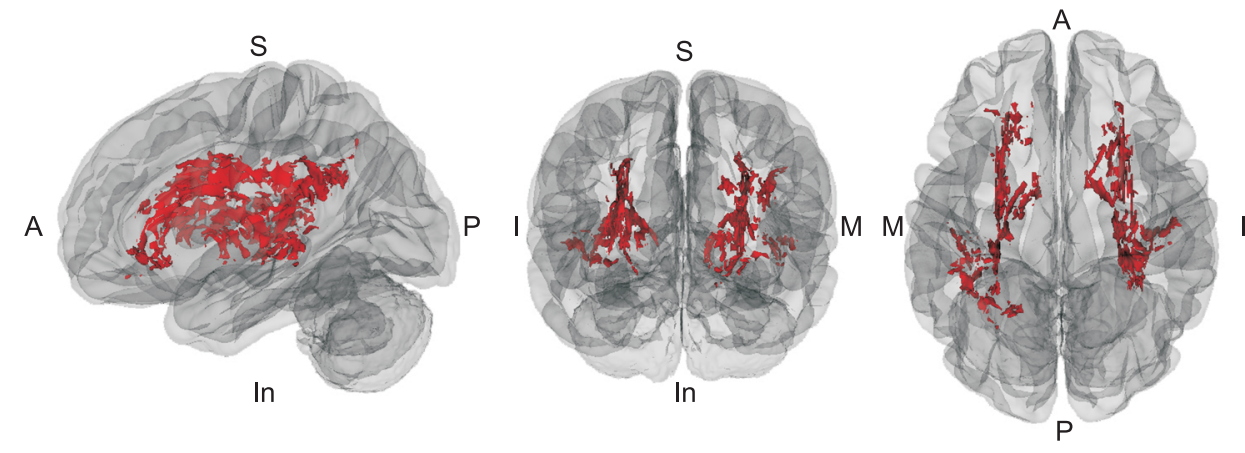

Fig. 1. Mapping of axial diffusivity (AD) in patients vs. controls. The images show the distributions of white matter structures in which the AD values were higher in patients with phantom limb pain than in healthy controls without pain, adjusted for depressive symptoms. A: anterior, P: posterior, S: superior, In: inferior, I: hemisphere associated with intact hand, M: hemisphere associated with missing hand. 

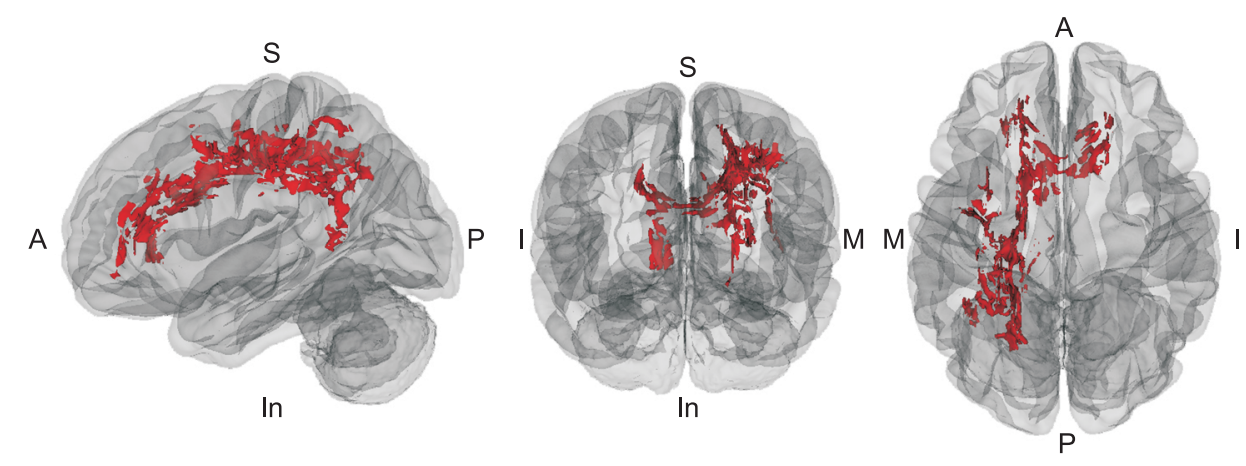

Fig. 2. Mapping of radial diffusivity (RD) correlates by phantom limb pain severity in the patient group. The distribution of white matter structures in which RD values were positively correlated with visual analogue scale scores in patients with phantom limb pain, when the Hamilton depression rating scale scores were included as a nuisance covariate. A: anterior, P: posterior, S: superior, In: inferior, I: hemisphere associated with intact hand, M: hemisphere associated with missing hand.
Table 3. Labels of White Matter Structures in Which the Radial Diffusivity Values Were Positively Correlated with VAS Scores in Patients with Phantom Limb Pain

\begin{tabular}{lrrr}
\hline \multicolumn{1}{c}{ White matter label (M or I) } & $\begin{array}{r}\text { Maximum } \\
t \text { value }\end{array}$ & $P$ value & $\begin{array}{r}\text { Ratio } \\
(\%)\end{array}$ \\
\hline Corpus callosum: genu & 8.4339 & 0.0401 & 8.90 \\
Corpus callosum: body & 8.3724 & 0.0395 & 15.49 \\
Posterior corona radiata (M) & 6.9882 & 0.0415 & 5.04 \\
Anterior corona radiata (M) & 4.8528 & 0.0416 & 9.63 \\
Superior corona radiata (M) & 4.8373 & 0.0370 & 7.27 \\
Superior longitudinal fasciculus (M) & 3.6407 & 0.0420 & 5.20 \\
Corpus callosum: splenium & 3.2276 & 0.0427 & 0.64 \\
Anterior corona radiata (I) & 2.7363 & 0.0473 & 1.53 \\
Cingulum (M) & 1.7956 & 0.0423 & 0.64 \\
Superior corona radiata (I) & 1.4948 & 0.0488 & 0.28 \\
Posterior thalamic radiation (M) & 0.1688 & 0.0427 & 0.04 \\
\hline
\end{tabular}

VAS: visual analogue scale, M: hemisphere associated with missing hand, I: hemisphere associated with intact hand.

\section{Discrimination of RD and AD measures}

The volume of white matter where the AD was different between two groups (Fig. 1) was $5,923 \mathrm{~mm}^{3}$, and the volume of white matter where the RD was correlated with the VAS scores (Fig. 2) was $4,965 \mathrm{~mm}^{3}$. Because the two derived measures of group comparison (Fig. 1) and of pain correlates (Fig. 2) were widespread in the hemispheres, we further discriminated the regions in which the two derived measures were overlaid. An overlapping area was identified in the corona radiata and had a volume of $155 \mathrm{~mm}^{3}$, corresponding to $2.6 \%$ and $3.1 \%$ of volumes 1 and 2 , respectively. Therefore, there was only minimal white matter in the corona radiata where the two derived measures intersected.

\section{DISCUSSION}

In this study we performed brain DTI analysis to examine the white matter features of patients with phantom limb pain after unilateral arm amputation. To our knowledge, this study is the first to show white matter alterations in the pain network induced by phantom limb pain. Moreover, this analysis of structural connectivity by DTI complements our previous study, proving that CBV change was associated with the emotional domain of the pain network in these patients [11].

DTI can be used to analyze white matter integrity and connectivity, effectively reconstructing the threedimensional distribution of the white matter pathways [1]. DTI parameters $\mathrm{MD}, \mathrm{AD}, \mathrm{RD}$, and FA are derived from the three eigenvalues $\left(\lambda_{1}, \lambda_{2}\right.$, and $\left.\lambda_{3}\right)$ calculated after generating the diffusion tensor from a series of diffusion-weighted images [20]. In turn, these DTI measures are highly sensitive to white matter pathology, and specific changes in these measures can be used to characterize the tissue microstructure [21]. A decrease in FA value indicates a loss of white matter integrity [21]. AD can be used to assess axonal function [22]. Increased RD is associated with demyelination, neuroinflammation with edema, or macrophage infiltration [23-25].

In this study, changes in the AD and pain correlates of the RD indicated significant structural connectivity changes in the white matter of patients with phantom limb pain. There are two broad considerations; first, patients with phantom limb pain had broad white matter deterioration, as reflected by the increased AD values and preserved RD values in the voxels of both hemispheres compared with those of controls. White matter injury is generally associated with increased $\mathrm{AD}$ and $\mathrm{RD}$ values, with the latter typically being more pronounced than the former [26]. Alterations in AD vary with the type of disease and its status, being reported to decrease over time in Huntington's disease [27], unchanged in amyotrophic lateral sclerosis [28], and increase in aging [29], Huntington's disease [30,31], Alzheimer's disease [32,33], Friedreich's ataxia [26], human immunodeficiency virus infection [34], and Gulf War illness [35]. The increase in AD value may represent increased extracellular water content secondary to axonal atrophy as a result of chronic injury, 
contrasting with decreased AD observed in acute injury [34]. Given that durations of phantom limb pain were 16 to 115 months, we conclude that the examination was sufficiently long enough after amputation for the AD to have increased for this reason. But it should also be noted that explanations about elevated AD may not be thorough, due to the technical limitations of DTI, although TBSS, as used in this study, has been presented as an approach for improving the sensitivity of DTI derived measures.

There is a need to discuss whether increased AD was associated with phantom limb pain or other conditions involved after amputation, because we did not enroll amputees without phantom limb pain as a control group. To exclude the effects of other conditions on the pain network, we examined the depressive mood and cognitive function, and given that HDRS scores were much higher in patients than in controls and that individual differences in the severity of depression may affect both the white matter microstructure [16] and pain [17], HDRS scores were included as a nuisance covariate in the statistical models, and we found the diffuse and symmetric increase of AD in the bilateral hemisphere. Considering the white matters in which $\mathrm{AD}$ increased, the pain and motor networks were involved; therefore, phantom limb pain as well as missing motion following amputation may have affected the diffusivity change in the brain.

Previous studies have indicated that white matter change was induced in the corpus callosum $[36,37]$ and bilateral hemispheres [38], even in unilateral limb amputation patients without phantom limb pain. The corpus callosum showed increased diffusivity and decreased FA in unilateral leg amputees compared with those in healthy controls [36,37]. Jiang et al. [38] demonstrated that unilateral leg amputees exhibited decreased FA in the white matter underlying the premotor cortex of the contralateral hemisphere to the amputation. In addition, the amputees also showed a decreased FA in the superior corona radiata and white matter regions underlying the temporal lobe of the ipsilateral hemisphere to the amputation. These studies suggest that unilateral limb amputation without phantom limb pain can lead to changes in the bilateral hemispheres through interhemispheric interactions.

The RD values were positively correlated with pain severity in the corpus callosum and white matter in the contralateral hemisphere to the missing hand. We confirmed that there was a little overlap between the white matter in which $A D$ values increased and that in which $R D$ values correlated with pain severity. We examined the percentage of amputation and the duration of phantom limb pain, in addition to pain intensity, and found that only pain intensity was related with increased $\mathrm{RD}$ values in the corpus callosum and white matter in the contralateral hemi- sphere to the missing hand. Therefore, it is reasonable to assume that phantom limb pain was associated with white matter integrity in the corpus callosum and the contralateral hemisphere to the missing hand.

Chronic pain and white matter in the brain are related, with typical examples being that central demyelinating disorders like multiple sclerosis [39] and trigeminal neuralgia [40] often induce pain. Moreover, chronic pain originating from organs other than the brain can induce various changes in white matter diffusivity. For example, patients with chronic recurring visceral pain from irritable bowel syndrome have been shown to display differential changes in the axons and myelin of the pain network [41]. In this study, patients had lower FA values in the thalamus, basal ganglia, and sensory/motor association regions, as well as higher FA values in the frontal lobe and corpus callosum. Patients also had reduced MD values in the globus pallidus, and increased MD values in the thalamus, internal capsule, and corona radiata. In patients with fibromyalgia, FA and MD have been reported to change unevenly in pain network structures $[42,43]$. Together, these findings suggest that it is reasonable to assume that chronic pain induces white matter changes, and includes both axonopathy and myelinopathy in various brain areas.

The patterns of increased diffusivity, and the correlation between diffusivity and pain intensity, were very similar with a previous CBV study [11]. We observed increased $\mathrm{CBV}$ in the ACC and OFC of both hemispheres regardless of the amputation side, which are regions associated with emotion in the cerebral pain network. We concluded that phantom limb pain following unilateral arm amputation was associated with plasticity of both the cortex and the linking white matter in the pain networks of both hemispheres. In addition, correlations between pain severity and CBV occurred in the ACC and OFC of the hemisphere connected to the missing hand. White matter changes identified in the present study could offer clues to the mechanism underlying the CBV changes observed in our previous case-control study of the same participants [11].

In that study, we did not investigate the changes in patients who had undergone amputation following both electrical and non-electrical injury, making it difficult to determine whether the observed changes in CBV were induced by electrical injury or phantom limb pain. However, the present results indicate that the changes in CBV were probably related to the phantom limb pain, as with the white matter change. Given the findings of the present DTI study and the previous CBV study, we considered that phantom limb pain was associated with diffuse changes in the pain network, and that these changes might have been the origin of the intractable pain experienced by our patients. 
This study had several limitations. First, this study was supplementary to our previous CBV study, so was also limited by the small sample size (ten patients). This made it difficult to identify patients with homogenous amputation characteristics, and will have weakened the statistical power. However, the current study does take a promising new step in improving our understanding of the mechanisms underlying phantom limb pain. Future studies should include a larger sample and should seek to demonstrate simultaneous changes in the gray and white matter with improved accuracy. Second, the authors assume that white matter changes were related with the phantom limb pain; however, we did not include amputees without phantom limb pain as a control group. We tried to exclude factors associated with amputation that may have effect on brain plasticity; however, there is a still a possibility that any factor had an effect on the increased white diffusivity. The third limitation is concerned with the acquisition of DTI data in this study, specifically a low magnetic field, large slice thickness, and a small number of diffusion directions. These limitations contrast with recent studies using DTI data and may have impaired our ability to extract sufficient directional information from the white matter. The fourth limitation is the small possibility that opioid-induced neurotoxicity might have had an effect on the white matter diffusivity. Though, some patients in this study were taking an opioid (oxycodone, maximal dose: $80 \mathrm{mg} /$ day or hydrocodone, maximal dose: $30 \mathrm{mg} /$ day) for pain control, the opioid-induced neurotoxicity tends to occur in frail older adults and those with renal failure. A patient who has been receiving a stable dose of an opioid is unlikely to develop opioid-induced neurotoxicity unless dehydration, infection, or a drug interaction has precipitated it [44].

In conclusion, we suggest that structural changes in the neural networks were consequences of phantom limb pain, manifesting as changes in the diffusivity of the pain network. The clinical significance of this study is that we need to consider central mechanisms for the treatment of phantom limb pain, particularly not only the cerebral hemisphere connected to the missing hand but also the cerebral hemisphere connected to the intact hand, because the phantom limb pain after unilateral arm amputation induced structural changes in the white matter of the bilateral hemisphere. For example, repetitive transcranial magnetic stimulation has been used to treat central neuropathic pain by modulating the pain network in brain [45]. Neural network modulation is determined by specific stimulation frequencies; low frequency stimulation of less than $1 \mathrm{~Hz}$ produces inhibition, whereas high frequency stimulation of more than $5 \mathrm{~Hz}$ produces excitation. Therefore, if we apply repetitive transcranial magnetic stimula- tion for the treatment of phantom pain, we will have to consider complex mechanisms, including which hemisphere should be stimulated and whether the hemisphere be inhibited or stimulated. In an upcoming study, we aim to examine DTI-MRI and CBV-MRI in patients with phantom limb pain after bilateral arm amputation to compare the differences between unilateral and bilateral pain network activation. This may provide further insights into the changes in the pain network after ceasing sensory and motor inputs.

\section{ACKNOWLEDGMENTS}

This research was supported by the Basic Science Research Program through the National Research Foundation of Korea (NRF), funded by the Ministry of Science, ICT \& Future Planning (2014R1A1A1006893), the Korea Health Technology R\&D Project through the Korea Health Industry Development Institute (KHIDI), funded by the Ministry of Health \& Welfare, Republic of Korea (HI15C1486020015), and the Hallym University Research Fund 2013 and 2014 (HURF-2013-29 and HURF-2014-06).

\section{CONFLICT OF INTEREST}

No potential conflict of interest relevant to this article was reported.

\section{ORCID}

Cheong Hoon Seo, https://orcid.org/0000-0003-4091-6382 Chang-hyun Park, https://orcid.org/0000-0001-5575-1467 Myung Hun Jung, https://orcid.org/0000-0003-2393-3930 Seungki Baek, https://orcid.org/0000-0002-3974-5822 Jimin Song, https://orcid.org/0000-0003-0853-0463 Eunsil Cha, https://orcid.org/0000-0003-3016-3508 Suk Hoon Ohn, https://orcid.org/0000-0002-1139-1946

\section{REFERENCES}

1. Wang Y, Liu G, Hong D, Chen F, Ji X, Cao G. White matter injury in ischemic stroke. Prog Neurobiol 2016; 141: 45-60.

2. Villain N, Fouquet M, Baron JC, Mézenge F, Landeau B, de La Sayette V, et al. Sequential relationships between grey matter and white matter atrophy and brain metabolic abnormalities in early Alzheimer's disease. Brain 2010; 133: 3301-14.

3. Manning KY, Schranz A, Bartha R, Dekaban GA, Barreira C, Brown A, et al. Multiparametric MRI changes persist beyond 
recovery in concussed adolescent hockey players. Neurology 2017; 89: 2157-66.

4. Arvanitakis Z, Fleischman DA, Arfanakis K, Leurgans SE, Barnes LL, Bennett DA. Association of white matter hyperintensities and gray matter volume with cognition in older individuals without cognitive impairment. Brain Struct Funct 2016; 221: 2135-46.

5. Willoch F, Rosen G, Tölle TR, Oye I, Wester HJ, Berner N, et al. Phantom limb pain in the human brain: unraveling neural circuitries of phantom limb sensations using positron emission tomography. Ann Neurol 2000; 48: 842-9.

6. Draganski B, Moser T, Lummel N, Gänssbauer S, Bogdahn U, Haas F, et al. Decrease of thalamic gray matter following limb amputation. Neuroimage 2006; 31: 951-7.

7. Flor H. Maladaptive plasticity, memory for pain and phantom limb pain: review and suggestions for new therapies. Expert Rev Neurother 2008; 8: 809-18.

8. May A. Chronic pain may change the structure of the brain. Pain 2008; 137: 7-15.

9. Makin TR, Scholz J, Henderson Slater D, Johansen-Berg H, Tracey I. Reassessing cortical reorganization in the primary sensorimotor cortex following arm amputation. Brain 2015; 138 Pt 8: 2140-6.

10. Vogt BA. Pain and emotion interactions in subregions of the cingulate gyrus. Nat Rev Neurosci 2005; 6: 533-44.

11. Seo CH, Park CH, Jung MH, Jang S, Joo SY, Kang Y, et al. Preliminary investigation of pain-related changes in cerebral blood volume in patients with phantom limb pain. Arch Phys Med Rehabil 2017; 98: 2206-12.

12. Assaf Y, Pasternak O. Diffusion tensor imaging (DTI)-based white matter mapping in brain research: a review. J Mol Neurosci 2008; 34: 51-61.

13. Neil JJ. Diffusion imaging concepts for clinicians. J Magn Reson Imaging 2008; 27: 1-7.

14. Yi JS, Bae SO, Ahn YM, Park DB, Noh KS, Shin HK, et al. Validity and reliability of the Korean Version of the Hamilton Depression Rating Scale (K-HDRS). J Korean Neuropsychiatr Assoc 2005; 44: 456-65.

15. Smith SM, Jenkinson M, Johansen-Berg H, Rueckert D, Nichols TE, Mackay CE, et al. Tract-based spatial statistics: voxelwise analysis of multi-subject diffusion data. Neuroimage 2006; 31: 1487-505.

16. Kieseppä T, Eerola M, Mäntylä R, Neuvonen T, Poutanen VP, Luoma $\mathrm{K}$, et al. Major depressive disorder and white matter abnormalities: a diffusion tensor imaging study with tractbased spatial statistics. J Affect Disord 2010; 120: 240-4.

17. Lindsay PG, Wyckoff M. The depression-pain syndrome and its response to antidepressants. Psychosomatics 1981; 22: 571-3, 576-7.

18. Winkler AM, Ridgway GR, Webster MA, Smith SM, Nichols TE. Permutation inference for the general linear model. Neuroimage 2014; 92: 381-97.
19. Smith SM, Nichols TE. Threshold-free cluster enhancement: addressing problems of smoothing, threshold dependence and localisation in cluster inference. Neuroimage 2009; 44: 83-98.

20. Song SK, Sun SW, Ju WK, Lin SJ, Cross AH, Neufeld AH. Diffusion tensor imaging detects and differentiates axon and myelin degeneration in mouse optic nerve after retinal ischemia. Neuroimage 2003; 20: 1714-22.

21. Alexander AL, Lee JE, Lazar M, Field AS. Diffusion tensor imaging of the brain. Neurotherapeutics 2007; 4: 316-29.

22. Mac Donald CL, Dikranian K, Bayly P, Holtzman D, Brody D. Diffusion tensor imaging reliably detects experimental traumatic axonal injury and indicates approximate time of injury. J Neurosci 2007; 27: 11869-76.

23. Budde MD, Janes L, Gold E, Turtzo LC, Frank JA. The contribution of gliosis to diffusion tensor anisotropy and tractography following traumatic brain injury: validation in the rat using Fourier analysis of stained tissue sections. Brain 2011; 134 Pt 8: 2248-60.

24. Kumar R, Woo MA, Macey PM, Fonarow GC, Hamilton MA, Harper RM. Brain axonal and myelin evaluation in heart failure. J Neurol Sci 2011; 307: 106-13.

25. Harrison DM, Shiee N, Bazin PL, Newsome SD, Ratchford JN, Pham D, et al. Tract-specific quantitative MRI better correlates with disability than conventional MRI in multiple sclerosis. J Neurol 2013; 260: 397-406.

26. Della Nave R, Ginestroni A, Diciotti S, Salvatore E, Soricelli A, Mascalchi M. Axial diffusivity is increased in the degenerating superior cerebellar peduncles of Friedreich's ataxia. Neuroradiology 2011; 53: 367-72.

27. Weaver KE, Richards TL, Liang O, Laurino MY, Samii A, Aylward EH. Longitudinal diffusion tensor imaging in Huntington's disease. Exp Neurol 2009; 216: 525-9.

28. Cosottini M, Giannelli M, Siciliano G, Lazzarotti G, Michelassi MC, Del Corona A, et al. Diffusion-tensor MR imaging of corticospinal tract in amyotrophic lateral sclerosis and pro-gressive muscular atrophy. Radiology 2005; 237: 258-64.

29. Sullivan EV, Rohlfing T, Pfefferbaum A. Quantitative fiber tracking of lateral and interhemispheric white matter systems in normal aging: relations to timed performance. Neurobiol Aging 2010; 31: 464-81.

30. Della Nave R, Ginestroni A, Tessa C, Giannelli M, Piacentini S, Filippi M, et al. Regional distribution and clinical correlates of white matter structural damage in Huntington disease: a tract-based spatial statistics study. AJNR Am J Neuroradiol 2010; 31: 1675-81.

31. Rosas HD, Lee SY, Bender AC, Zaleta AK, Vangel M, Yu P, et al. Altered white matter microstructure in the corpus callosum in Huntington's disease: implications for cortical "disconnection". Neuroimage 2010; 49: 2995-3004.

32. Acosta-Cabronero J, Williams GB, Pengas G, Nestor PJ. Absolute diffusivities define the landscape of white matter degen- 
eration in Alzheimer's disease. Brain 2010; 133 Pt 2: 529-39.

33. Salat DH, Tuch DS, van der Kouwe AJ, Greve DN, Pappu V, Lee SY, et al. White matter pathology isolates the hippocampal formation in Alzheimer's disease. Neurobiol Aging 2010; 31: 244-56.

34. Zhu T, Zhong J, Hu R, Tivarus M, Ekholm S, Harezlak J, et al. Patterns of white matter injury in HIV infection after partial immune reconstitution: a DTI tract-based spatial statistics study. J Neurovirol 2013; 19: 10-23.

35. Rayhan RU, Stevens BW, Timbol CR, Adewuyi O, Walitt B, VanMeter JW, et al. Increased brain white matter axial diffusivity associated with fatigue, pain and hyperalgesia in Gulf War illness. PLoS One 2013; 8: e58493. doi: 10.1371/journal. pone.0058493.

36. Li Z, Li C, Fan L, Jiang G, Wu J, Jiang T, et al. Altered microstructure rather than morphology in the corpus callosum after lower limb amputation. Sci Rep 2017; 7: 44780.

37. Simões EL, Bramati I, Rodrigues E, Franzoi A, Moll J, Lent $R$, et al. Functional expansion of sensorimotor representation and structural reorganization of callosal connections in lower limb amputees. J Neurosci 2012; 32: 3211-20.

38. Jiang G, Yin X, Li C, Li L, Zhao L, Evans AC, et al. The plasticity of brain gray matter and white matter following lower limb amputation. Neural Plast 2015; 2015: 823185.

39. Moulin DE. Pain in central and peripheral demyelinating disorders. Neurol Clin 1998; 16: 889-98.

40. Love S, Hilton DA, Coakham HB. Central demyelination of the Vth nerve root in trigeminal neuralgia associated with vascular compression. Brain Pathol 1998; 8: 1-11.

41. Ellingson BM, Mayer E, Harris RJ, Ashe-McNally C, Naliboff $\mathrm{BD}$, Labus JS, et al. Diffusion tensor imaging detects microstructural reorganization in the brain associated with chronic irritable bowel syndrome. Pain 2013; 154: 1528-41.

42. Lutz J, Jäger L, de Quervain D, Krauseneck T, Padberg F, Wichnalek $\mathrm{M}$, et al. White and gray matter abnormalities in the brain of patients with fibromyalgia: a diffusion-tensor and volumetric imaging study. Arthritis Rheum 2008; 58: 3960-9.

43. Sundgren PC, Petrou M, Harris RE, Fan X, Foerster B, Mehrotra N, et al. Diffusion-weighted and diffusion tensor imaging in fibromyalgia patients: a prospective study of whole brain diffusivity, apparent diffusion coefficient, and fraction anisotropy in different regions of the brain and correlation with symptom severity. Acad Radiol 2007; 14: 839-46.

44. Gallagher R. Opioid-induced neurotoxicity. Can Fam Physician 2007; 53: 426-7.

45. Ohn SH, Chang WH, Park CH, Kim ST, Lee JI, Pascual-Leone A, et al. Neural correlates of the antinociceptive effects of repetitive transcranial magnetic stimulation on central pain after stroke. Neurorehabil Neural Repair 2012; 26: 344-52. 